\title{
Influence of complex configurations on properties of pygmy dipole resonance in neutron-rich $\mathrm{Ca}$ isotopes
}

\author{
N. N. Arsenyev, ${ }^{1}$ A. P. Severyukhin,,${ }^{1,2}$ V. V. Voronov, ${ }^{1}$ and Nguyen Van Giai ${ }^{3}$ \\ ${ }^{1}$ Bogoliubov Laboratory of Theoretical Physics, Joint Institute for Nuclear Research, 141980 Dubna, Moscow region, Russia \\ ${ }^{2}$ Dubna State University, 141982 Dubna, Moscow region, Russia \\ ${ }^{3}$ Institut de Physique Nucléaire, CNRS-IN2P3 and Univ. Paris-Sud, 91405 Orsay, France
}

(Dated: September 20, 2018)

\begin{abstract}
Starting from the quasiparticle random phase approximation based on the Skyrme interaction SLy5, we study the effects of phonon-phonon coupling (PPC) on the low-energy electric dipole response in ${ }^{40-58} \mathrm{Ca}$. Using the same set of parameters we describe available experimental data for ${ }^{40,44,48} \mathrm{Ca}$ and give prediction for ${ }^{50-58} \mathrm{Ca}$. The inclusion of the PPC results in the formation of low-energy $1^{-}$states. There is an impact of the PPC effect on low-energy $E 1$ strength of ${ }^{40,44,48} \mathrm{Ca}$. The PPC effect on the electric dipole polarizability is discussed. We predict a strong increase of the summed $E 1$ strength below $10 \mathrm{MeV}$, with increasing neutron number from ${ }^{48} \mathrm{Ca}$ till ${ }^{58} \mathrm{Ca}$.
\end{abstract}

PACS numbers: 21.60.Jz, 21.10.Pc, 21.30.Fe, 27.40.+z

\section{INTRODUCTION}

Collective dipole excitations are a common phenomenon of finite fermion systems. In atomic nuclei they can arise, for instance, from out-of-phase oscillations of the proton and neutron "fluids" giving the well known giant dipole resonance (GDR) [1]. Systematic investigations established it to be a global feature of nuclei from the very light to the heaviest nuclei [2, 3]. In recent years, the interest is more focused on the low-lying dipole strength, that is located below the GDR energies. The concentration of the $E 1$ strength around the particle separation energy is commonly called the pygmy dipole resonance (PDR) because of its weak strength in comparison with the GDR, which dominates the $E 1$ strength distribution in nuclei [4]. In analogy to the GDR, the PDR has been interpreted as a collective oscillation of the neutron skin with respect to a $N \approx Z$ inert core (see Ref. [5] and references therein). The total sum of the measured energy-weighted sum rule of such $E 1$ distributions is less than $1-2 \%$ of the Thomas-Reiche-Kuhn (TRK) sum rule value for stable nuclei and less than 5-6\% for unstable neutron-rich nuclei [4]. Recent theoretical calculations indicate that such a low-energy peak is a common property of neutron-rich nuclei lying in different mass regions [6, 7]. The occurrence of non-negligible low-lying $E 1$-strength can influence the radiative neutron capture cross section by orders of magnitude and, consequently, also the rate of the astrophysical $r$-process nucleosynthesis [8]. The PDR study is expected to provide information on the symmetry energy term of the nuclear equation of state [9, 10].

The strong proton shell closure at $Z=20$ and the already good experimental knowledge of the chain of calcium isotopes makes [11-14] calcium an attractive clement for a PDR study. Indeed, indications of a trend for increasing low-energy dipole strength with increasing mass can be observed in the dipole excitation functions (above neutron separation energy) in the stable $\mathrm{Ca}$ iso- topes [11, 12]. The results were generally consistent with the theoretical prediction regarding the shifting of dipole strength to lower energies, see, e.g., Refs. [6, 15 17]. Moreover, recent experimental studies indicate $N=32$ as a new magic number in $\mathrm{Ca}$ isotopes due to the high energy of the first $2^{+}$state in ${ }^{52} \mathrm{Ca}$ [18] and the trend obtained for the two-neutron separation energies [19]. The first experimental spectroscopic study of low-lying states in ${ }^{54} \mathrm{Ca}$ have been performed at RIKEN [20]: the $2_{1}^{+}$ energy in ${ }^{54} \mathrm{Ca}$ was found to be only $\sim 500 \mathrm{keV}$ below that in ${ }^{52} \mathrm{Ca}$, suggesting a $N=34$ new shell closure. Finally, we note that new progress in the production of neutron-rich $\mathrm{Ca}$ isotopes can be expected at the NSCL at Michigan State University [21]. Future measurements of excited states and masses for the neighboring Ca isotopes will further enhance our understanding of nuclear states in very neutron-rich systems. Thus, the spectroscopy of neutron-rich calcium isotopes provides a valuable information, with important tests of theoretical calculations.

A powerful microscopic approach is the quasiparticlephonon model (QPM) 22]. Its ability for the describing the low-energy nuclear spectroscopy has recently been reviewed in Ref. [23]. The model Hamiltonian is diagonalized in a space spanned by states composed of one, two and three phonons which are generated in quasiparticle random phase approximation (QRPA) [24, 25]. The separable form of the residual interaction is the practical advantage of the QPM which allows one to perform the structure calculations in large configurational spaces. The mean field is modelized by a Woods-Saxon potential well. These are the basic ingredients of the QPM. The single-particle energies and ground-state properties in general are critical quantities for extrapolations of QRPA and QPM calculations into unknown mass regions. A special emphasis on a reliable description of the meanfield part, reproducing as closely as possible the groundstate properties of nuclei along an isotopic chain has been done in [26, 27]. This is achieved by solving the groundstate problem in a semi-microscopic approach based on a Skyrme energy density functional (EDF) [26, 27]. The 
$\mathrm{EDF}+\mathrm{QPM}$ calculations have been applied for calculating the low-energy dipole strength [26, 27], as well as used for astrophysical applications [28]. The results indicate that the radiative capture cross sections are underestimated by a factor of about two by the QRPA for the $N=50$ nuclei. This sensitivity is the cause of the importance of the multiphonon coupling and of the relevance of the EDF+QPM approach for astrophysical applications.

The QRPA with a self-consistent mean-field derived from Skyrme EDF is one of the most successful methods for studying the low-energy dipole strength, see e.g., [16, 29 32]. Such an approach describes the properties of the low-lying states less accurately than more phenomenological ones, but the results are in a reasonable agreement with experimental data. On the other hand, due to the anharmonicity of vibrations there is a coupling between one-phonon and more complex states [22, 23]. The main difficulty is that the complexity of calculations beyond standard QRPA increases rapidly with the size of the configuration space, so that one has to work within limited spaces. Using a finite-rank separable approximation (FRSA) [33 36] for the residual interaction one can overcome this difficulty. Alternative schemes to factorize the residual Skyrme interaction have also been considered in Refs. [37 39]. The FRSA was thus used to study the electric low-energy excitations and giant resonances within and beyond the QRPA [35, 36, 40, 41]. In this paper our approach applied for PDR features of neutron-rich nuclei. We will give an illustration of our approach for ${ }^{48} \mathrm{Ca}$ with closed neutron shell in comparison to the $N=30$ isotope ${ }^{50} \mathrm{Ca}$. Preliminary results of our studies for neutron-rich $\mathrm{Sn}$ isotopes are reported in Refs. [31, 42, 43].

The paper is organized as follows. A brief summary of the formalism including the effects of the phonon-phonon coupling is given in Sec. II. Some details about the numerical calculations are presented in Sec. III, while in Sec. IV, results are analyzed and compared with available experimental data. Finally, our conclusions are laid in the last section.

\section{THE FRSA MODEL}

The starting point of the method is the HartreeFock (HF)-BCS calculation [44] of the ground state based on Skyrme interactions. Spherical symmetry is imposed on the quasiparticle wave functions. The continuous part of the single-particle spectrum is discretized by diagonalizing the Skyrme HF Hamiltonian on a harmonic oscillator basis. In the particle-hole (p-h) channel we use the Skyrme interaction with the tensor components and their inclusion leads to the modification of the spin-orbit potential [45, 46]. The pairing correlations are generated by a density-dependent zero-range force

$$
V_{\text {pair }}\left(\mathbf{r}_{1}, \mathbf{r}_{2}\right)=V_{0}\left[1-\eta\left(\frac{\rho\left(r_{1}\right)}{\rho_{0}}\right)^{\gamma}\right] \delta\left(\mathbf{r}_{1}-\mathbf{r}_{2}\right),
$$

where $\rho\left(r_{1}\right)$ is the particle density in coordinate space, $\rho_{0}$ being the nuclear matter saturation density; $\gamma, \eta$, and $V_{0}$ are adjusted parameters. The parameters are determined by adjusting the empirical odd-even mass differences of the nuclei in the region under study.

To build the QRPA equations on the basis of HF-BCS quasiparticle states, the residual interaction is consistently derived from the Skyrme force in the p-h channel and from the zero-range pairing force in the particleparticle (p-p) channel [4]]. The FRSA for the residual interaction enables us to find QRPA eigenvalues as the roots of a relatively simple secular equation 33,36 . The cut-off of the discretized continuous part of the singleparticle spectra is at the energy of $100 \mathrm{MeV}$. This is sufficient to exhaust practically all the sum rules and, in particular, the TRK sum rule with the enhancement factor $\kappa$ for the EDF. The FRSA is discussed in more details in Refs. 36, 41, 48.

To take into account the effects of the phonon-phonon coupling (PPC) we follow the basic QPM ideas [22, 23]. We construct the wave functions from a linear combination of one-phonon and two-phonon configurations

$$
\begin{array}{r}
\Psi_{\nu}(\lambda \mu)=\left(\sum_{i} R_{i}(\lambda \nu) Q_{\lambda \mu i}^{+}\right. \\
\left.+\sum_{\lambda_{1} i_{1} \lambda_{2} i_{2}} P_{\lambda_{2} i_{2}}^{\lambda_{1} i_{1}}(\lambda \nu)\left[Q_{\lambda_{1} \mu_{1} i_{1}}^{+} Q_{\lambda_{2} \mu_{2} i_{2}}^{+}\right]_{\lambda \mu}\right)|0\rangle,
\end{array}
$$

where $\lambda$ denotes the total angular momentum and $\mu$ its z-projection in the laboratory system. The ground state is the QRPA phonon vacuum $|0\rangle$. The unknown amplitudes $R_{i}(\lambda \nu)$ and $P_{\lambda_{2} i_{2}}^{\lambda_{1} i_{1}}(\lambda \nu)$ are determined from the variational principle, which leads to a set of linear equations [35, 41]

$$
\begin{array}{r}
\left(\omega_{\lambda i}-E_{\nu}\right) R_{i}(\lambda \nu) \\
+\sum_{\lambda_{1} i_{1} \lambda_{2} i_{2}} U_{\lambda_{2} i_{2}}^{\lambda_{1} i_{1}}(\lambda i) P_{\lambda_{2} i_{2}}^{\lambda_{1} i_{1}}(\lambda \nu)=0 \\
2\left(\omega_{\lambda_{1} i_{1}}+\omega_{\lambda_{2} i_{2}}-E_{\nu}\right) P_{\lambda_{2} i_{2}}^{\lambda_{1} i_{1}}(\lambda \nu) \\
+\sum_{i} U_{\lambda_{2} i_{2}}^{\lambda_{1} i_{1}}(\lambda i) R_{i}(\lambda \nu)=0 .
\end{array}
$$

The rank of the set of linear equations (3) and (4) is equal to the number of one- and two-phonon configurations included in the wave function (2). Its solution requires to compute the Hamiltonian matrix elements coupling one- and two-phonon configurations:

$$
U_{\lambda_{2} i_{2}}^{\lambda_{1} i_{1}}(\lambda i)=\left\langle 0\left|Q_{\lambda i} H\left[Q_{\lambda_{1} i_{1}}^{+} Q_{\lambda_{2} i_{2}}^{+}\right]_{\lambda}\right| 0\right\rangle .
$$

Equations (3) and (4) have the same form as the QPM equations 22, 23], where the single-particle spectrum and the residual interaction are derived from the same Skyrme EDF. 

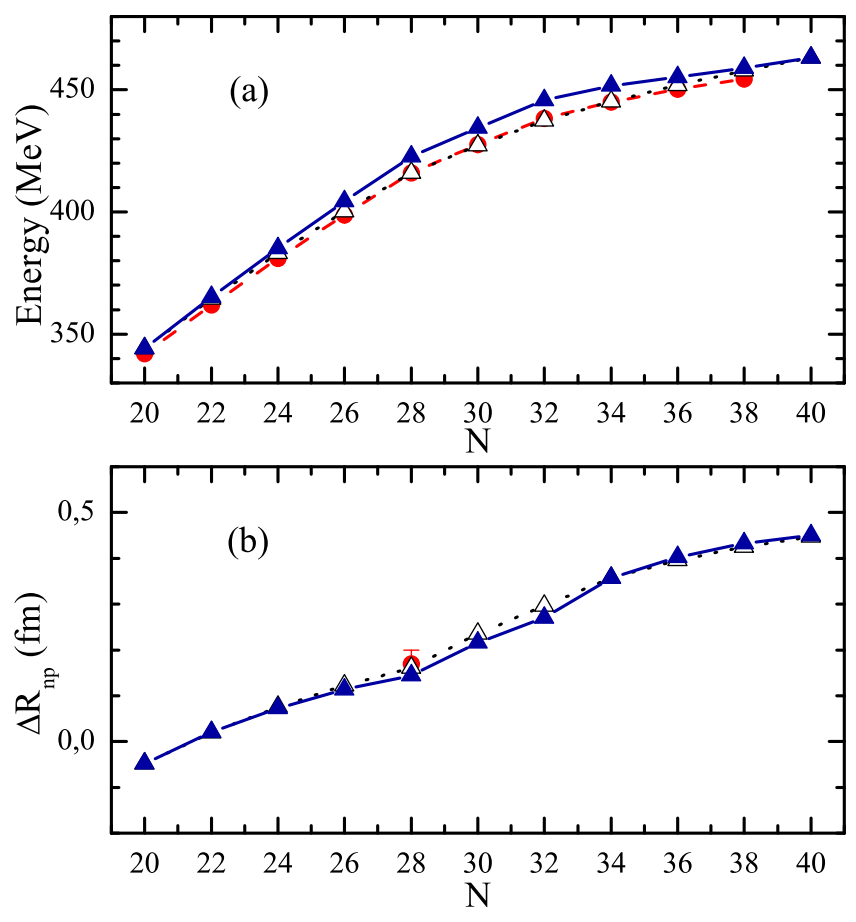

FIG. 1: (Color online) (a) Binding energies of the even-even $\mathrm{Ca}$ isotopes as a function of neutron number, compared with experiment and extrapolated energies (filled circles) from the AME2012 atomic mass evaluation [51]. Results of the calculations within the HF-BCS with the SLy5 EDF (open triangles) and with SLy5+T (filled triangles). (b) The neutron skin thickness $\Delta R_{n p}$ of the Ca isotopes calculated within the HFBCS approach with the SLy5 EDF (open triangles) and with SLy5+T (filled triangles). Experimental data of the neutron skin thickness are taken from Ref. [52] (filled circles).

\section{DETAILS OF CALCULATIONS}

We apply the above approach to study the influence of the PPC on the strength $E 1$ distributions of the neutron-rich $\mathrm{Ca}$ isotopes. We use the Skyrme interactions SLy5 [49] and SLy5+T [50] in the p-h channel. The parameters of the force SLy5 have been adjusted to reproduce nuclear matter properties, as well as nuclear charge radii, binding energies of doubly magic nuclei. The force $\mathrm{SLy} 5+\mathrm{T}$ involves the tensor terms added without refitting of the parameters of the central interaction (the tensor interaction parameters are $\alpha_{T}=-170 \mathrm{MeVfm}^{5}$ and $\beta_{T}=100 \mathrm{MeVfm}^{5}$ ). These parametrizations describe correctly the binding energies of even-even $\mathrm{Ca}$ isotopes. This is illustrated in Fig. 1(a), where the calculated binding energies for ${ }^{40-60} \mathrm{Ca}$ and the experimental and extrapolated data [51] are shown. The agreement between the SLy5 results and data is reasonable, the deviations being less than 1\%. On the other hand, comparing SLy5 and SLy5+T results shows that the maximum relative difference between the binding energies is about $2 \%$ for the case of ${ }^{52} \mathrm{Ca}$. In the case of the SLy5+T EDF, these deviations are connected with the central Skyrme param- eters which have not been refitted after including the tensor terms [50].

In Fig. 1 (b), we show the neutron skin thickness of $\mathrm{Ca}$ isotopes as a function of the neutron number. The neutron skin thickness $\Delta R_{n p}$ is defined as

$$
\Delta R_{n p}=\sqrt{\left\langle r^{2}\right\rangle_{n}}-\sqrt{\left\langle r^{2}\right\rangle_{p}}
$$

As can be seen from Fig. 1(b), the proton-neutron rms differences become larger when the neutron number increases. The same evolution is obtained with other Skyrme EDF's [6]. In the case of ${ }^{48} \mathrm{Ca}$, the experimental neutron skin thickness $(0.14-0.20 \mathrm{fm})$ has been determined from the $E 1$ strength distribution which is extracted from proton inelastic scattering [52]. HFBCS analysis gives the neutron skin $\Delta R_{n p}$ of ${ }^{48} \mathrm{Ca}$ to be $0.16 \mathrm{fm}$ and $0.14 \mathrm{fm}$ with the SLy5 and SLy5+T EDF's, respectively. The theoretical "model-averaged" estimate for $\Delta R_{n p}$ is $0.176 \pm 0.018 \mathrm{fm}$ [53]. In addition, the $a b$ initio calculations for the neutron skin in ${ }^{48} \mathrm{Ca}$ is $0.12 \leq \Delta R_{n p} \leq 0.15 \mathrm{fm}[54]$.

For the interaction in the p-p channel, we use a zerorange volume force, i.e., $\eta=0$ in Eq. (11). The strength $V_{0}$ is taken equal to $-270 \mathrm{MeVfm}^{3}$. This value of the pairing strength is fitted to reproduce the experimental neutron pairing energies of ${ }^{50,52,54} \mathrm{Ca}$ obtained from binding energies of neighboring isotopes. This choice of the pairing interaction has also been used for a satisfactory description of the experimental data of ${ }^{70,72,74,76} \mathrm{Ni}$ [48], ${ }^{90,92} \mathrm{Zr}$ and ${ }^{92,94}$ Mo [41]. Thus, hereafter we use the Skyrme interaction SLy5 with and without tensor components in the particle-hole channel together with the volume zerorange force acting in the particle-particle channel.

Valuable information about nuclear properties can be obtained from studies of the one-neutron $\left(S_{1 n}\right)$ and twoneutron $\left(S_{2 n}\right)$ separation energies. In addition, the separation energies are very important for the nuclear shell structure. Therefore, it is interesting to further investigate the evolution of one- and two-neutron separation energies with both Skyrme interactions. The neutron separation energies are defined as

$$
S_{x n}=B(Z, N)-B(Z, N-X),
$$

The calculated $S_{1 n}$ and $S_{2 n}$ values in the even Ca isotopes are compared with the experimental data [51] (or values deduced from systematic trends) in Fig. 2 . Despite the overestimation of the Ca binding energies, the effective interactions SLy5 and SLy5+T give a reasonable reproduction of the experimental trends in stable nuclei. It should be noted that the binding energies of the odd $\mathrm{Ca}$ isotopes are calculated with the blocking effect for unpaired nucleons [55, 56]. For ${ }^{39} \mathrm{Ca}$, the neutron quasiparticle blocking is based on filling the $\nu 1 d \frac{3}{2}$ subshell while the $\nu 1 f \frac{7}{2}$ subshell should be blocked for ${ }^{41,43,45,47} \mathrm{Ca}$. The neutron $\nu 2 p \frac{3}{2}, \nu 2 p \frac{1}{2}$ and $\nu 1 f \frac{5}{2}$ subshells are chosen to be blocked in the cases of the ${ }^{49,51} \mathrm{Ca}$, ${ }^{53} \mathrm{Ca}$ and ${ }^{55,57,59} \mathrm{Ca}$ isotopes, respectively. The existing 


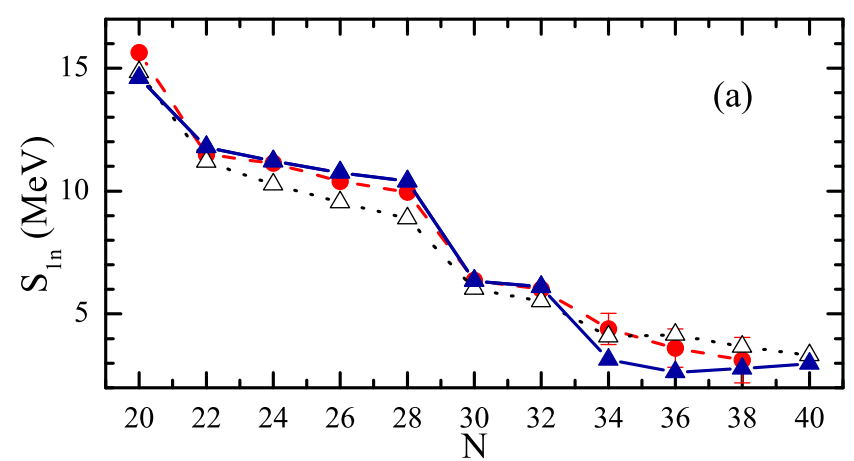

TABLE I: Energies and $B(E \lambda)$ values for up-transitions to the $\lambda_{1}^{\pi}$ states in ${ }^{46,48,50} \mathrm{Ca}$. Experimental data are taken from Refs. 60 63.

\begin{tabular}{cccccc}
\hline \hline & $\lambda_{1}^{\pi}$ & \multicolumn{2}{c}{$\begin{array}{c}\text { Energy } \\
(\mathrm{MeV})\end{array}$} & $\begin{array}{c}B\left(E \lambda ; 0_{g s}^{+} \rightarrow \lambda_{1}^{\pi}\right) \\
\left(\mathrm{e}^{2} \mathrm{~b}^{\lambda}\right)\end{array}$ \\
& & Expt. & Theory & Expt. & Theory \\
\hline${ }^{46} \mathrm{Ca}$ & $2_{1}^{+}$ & 1.346 & 2.05 & $0.0127 \pm 0.0023$ & 0.0070 \\
& $3_{1}^{-}$ & 3.614 & 4.57 & $0.006 \pm 0.003$ & 0.0049 \\
& $4_{1}^{+}$ & 2.575 & 2.30 & & 0.00035 \\
& $5_{1}^{-}$ & 4.184 & 4.67 & & 0.00027 \\
\hline${ }^{48} \mathrm{Ca}$ & $2_{1}^{+}$ & 3.832 & 3.19 & $0.00968 \pm 0.00105$ & 0.0065 \\
& $3_{1}^{-}$ & 4.507 & 4.47 & $0.0083 \pm 0.0020$ & 0.0038 \\
& $4_{1}^{+}$ & 4.503 & 3.51 & & 0.00035 \\
& $5_{1}^{-}$ & 5.729 & 4.52 & & 0.00026 \\
\hline${ }_{50} \mathrm{Ca}$ & $2_{1}^{+}$ & 1.027 & 1.50 & $0.00375 \pm 0.00010$ & 0.0018 \\
& $3_{1}^{-}$ & 3.997 & 4.36 & & 0.0045 \\
& $4_{1}^{+}$ & 4.515 & 3.75 & & 0.00051 \\
& $5_{1}^{-}$ & 5.110 & 4.45 & & 0.00029 \\
\hline \hline
\end{tabular}
panel) neutron separation energies for $\mathrm{Ca}$ isotopes as a function of neutron number, calculated with the SLy5 EDF (open triangles) and with SLy5+T (filled triangles). The experimental and extrapolated energies (filled circles) are from the AME2012 atomic mass evaluation [51].

experimental data show a different A-behavior, namely, the factor 5 reduction of $S_{1 n}$ values and the 7-time reduction of $S_{2 n}$ values from ${ }^{40} \mathrm{Ca}$ to ${ }^{58} \mathrm{Ca}$. In general, both the SLy5 and SLy5+T interactions give an excellent description of $S_{1 n}$ and $S_{2 n}$ for ${ }^{50,52} \mathrm{Ca}$ isotopes. Most notably, the major shell closure at the magic neutron number $N=20$ is too pronounced. Also, sharp decreases in separation energies are seen at the magic neutron number $N=28$. Significant differences are developed starting from ${ }^{52} \mathrm{Ca}$. In the case of the SLy5 interaction our calculations predicted a monotonic decrease of $S_{2 n}$ when going from ${ }^{52} \mathrm{Ca}$ to ${ }^{60} \mathrm{Ca}$. On the other hand, the presence of tensor components brings a drop in the theoretical $S_{2 n}$ in ${ }^{52,54} \mathrm{Ca}$ [57]. This corresponds to the hypothetical shell closures at $N=32,34$. This suggests that these nuclei are magic for the used interaction (see in Fig. 2(b)), thus matching predictions from shell model calculations including three-body forces [58, 59]. This view is supported by precision mass measurements [19, 20]. Thus, this jump is a shell effect, and the results indicate that the various forces lead to different detailed shell structures.

In order to construct the wave functions (2) of the $1^{-}$states, in the present study we take into account all two-phonon terms that are constructed from the phonons with multipolarities $\lambda \leq 5[31,42,43]$. As an example the energies and reduced transition probabilities of the first

$2^{+}, 3^{-}, 4^{+}$and $5^{-}$phonons for ${ }^{46,48,50} \mathrm{Ca}$ are presented in Table I. The QRPA results obtained with the SLy5 EDF are compared with the experimental data 60,63$]$. As one can see, the overall agreement of the energies and $B(E \lambda)$ values with the data looks reasonable. All dipole excitations with energies below $35 \mathrm{MeV}$ and 15 most collective phonons of the other multipolarities are included in the wave function (2). We have checked that extending the configuration space plays a minor role in our calculations.

It is interesting to examine the energies and transition probabilities of the first collective phonons which leads to the minimal two-phonon energy and the maximal matrix elements for coupling of the one- and two-phonon configurations (5). The calculated $2_{1}^{+}$energies and transition probabilities in the neutron-rich $\mathrm{Ca}$ isotopes are compared with existing experimental data $18,20,63$ in Fig. 3. The first $2^{+}$states of the even-even ${ }^{46-58} \mathrm{Ca}$ isotopes exhibit pure neutron two-quasiparticle (2QP) excitations $(>72 \%)$. It is worth mentioning that a similar observation has been found in Ref. [16], where the SkM* interaction was used. There is a marked increase of the $2_{1}^{+}$energy of ${ }^{48} \mathrm{Ca}$ in comparison with those in ${ }^{46} \mathrm{Ca}$ and ${ }^{50} \mathrm{Ca}$. It corresponds to a standard evolution of the $2_{1}^{+}$ energy near closed shells. It should be noted that including the tensor components changes the contributions of the main configurations only slightly, but the general structure of the $2_{1}^{+}$state remains the same. In the following, we analyze the PPC effects in the case of the first $2^{+}$excitations. In these calculations all two-phonon configurations below $20 \mathrm{MeV}$ are used. In ${ }^{46-58} \mathrm{Ca}$ isotopes, 


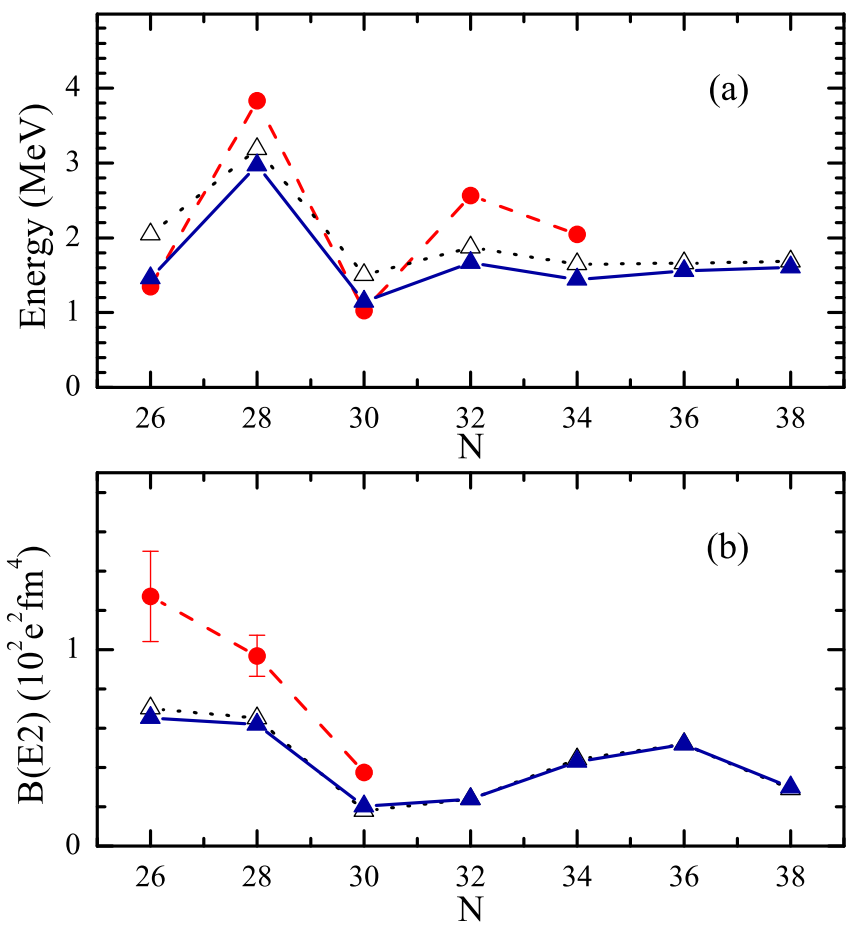

FIG. 3: (Color online) Energies (top panel) and $B(E 2)$ (lower panel) values for up-transitions to the $2_{1}^{+}$states in the neutron-rich $\mathrm{Ca}$ isotopes. Results of the calculations within the QRPA (open triangles) with the SLy5 EDF and the QRPA plus PPC (filled triangles) are shown. Experimental data (filled circles) are taken from Refs. [18, 20, 63].

the crucial contribution in the wave function structure of the first $2^{+}$state comes from the $\left[2_{1}^{+}\right]_{Q R P A}(>89 \%)$ configuration, i.e., $R_{i=1}^{2}(\lambda=2 \nu=1)>0.89$. Using the set of linear equations (3) and (4), one can get $E_{1} \leq \omega_{21}$. Since the transition matrix elements for direct excitation of two-phonon components from the ground state are about two orders of magnitude smaller as compared to the excitation of one-phonon components, it follows that $B(E 2)_{P P C} \leq B(E 2)_{Q R P A}$ in the case of the first $2^{+}$state. The decrease of the $2_{1}^{+}$energies and the $B(E 2)$ values is shown in Fig. 3 It is worth to mention that the first discussion of the PPC effect for the $2_{1}^{+}$properties based on QRPA calculations with Skyrme forces has been done in Ref. 35]. Fig. 3 demonstrates that the calculated energies and $B(E 2)$ values of the first $2^{+}$excited states deviate from the experimental data. In the case of ${ }^{46} \mathrm{Ca}$, the $B(E 2)$ value is about 1.5 times less than the data. This probably points to a particular problem due to the EDF used here rather than to a deficiency of our model space.

\section{RESULTS AND DISCUSSION}

As a first step in the present analysis, we examine the PPC effects on the E1 strength distributions for
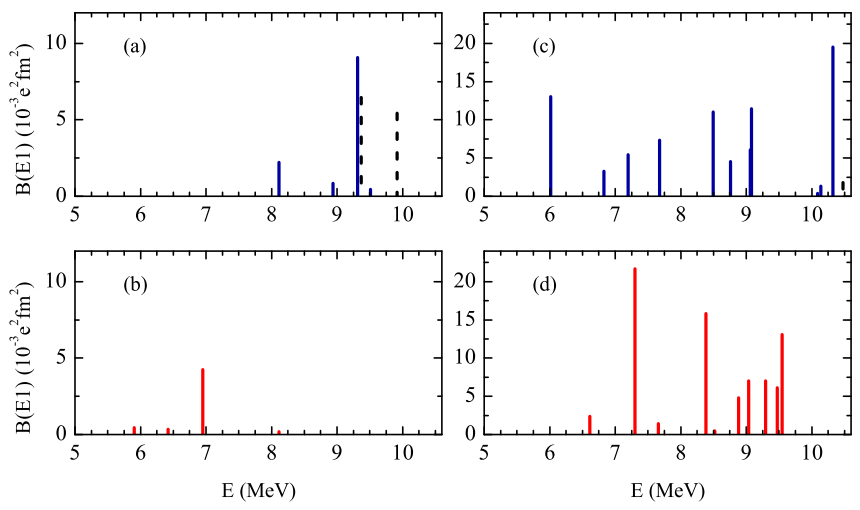

FIG. 4: (Color online) Low-energy $E 1$ strength distributions of ${ }^{40} \mathrm{Ca}$ (resp. ${ }^{48} \mathrm{Ca}$ ) are shown in the left (resp. right) panels. The dashed and solid lines correspond to the SLy5 calculations within the RPA and taking into account the PPC effects, respectively. Panels (b) and (d): experimental data are from Ref. [14].

doubly-magic ${ }^{40,48} \mathrm{Ca}$ isotopes. A comparison of such calculations with recent experimental data [14] demonstrates that the RPA approach cannot reproduce correctly the low-energy $E 1$ strength distributions, see Fig. 4. Let us discuss the properties of the lowest dipole state. For ${ }^{48} \mathrm{Ca}$ this state is mainly characterized by the two-phonon component $\left[2_{1}^{+} \otimes 3_{1}^{-}\right]$arising from the coupling between the first quadrupole and octupole phonons. We find a nice agreement with the data, where the candidate for the two-phonon $1^{-}$state is expected at an energy of $7.298 \mathrm{MeV}$ with $B\left(E 1 ; 0_{g s}^{+} \rightarrow 1_{1}^{-}\right)=$ $18.6 \pm 1.8 \times 10^{-3} \mathrm{e}^{2} \mathrm{fm}^{2}\left[11\right.$. For ${ }^{40} \mathrm{Ca}$ the PPC calculation predicts the first $1^{-}$state significantly higher than the experimental two-phonon candidate [64] (see Fig. 4). It is worth pointing out that our results for ${ }^{40,48} \mathrm{Ca}$ are in good agreement with the RQTBA calculations taking into account the effects of coupling between quasiparticles and phonons [17]. In addition, we discuss the GDR energy region. For ${ }^{48} \mathrm{Ca}$, the photo-absorption process is well studied experimentally. The photo-absorption cross section up to $27 \mathrm{MeV}$ is displayed in Fig. [5(a). The cross section is computed by using a Lorentzian smearing with an averaging parameter $\Delta=1.0 \mathrm{MeV}$. The PPC effects yield a noticeable redistribution of the GDR strength in comparison with the RPA results. It is worth mentioning that the coupling increases the GDR width from $6.9 \mathrm{MeV}$ to $7.3 \mathrm{MeV}$ in the energy region $10-26 \mathrm{MeV}$. Also, the PPC induces a $300 \mathrm{keV}$ downward shift of the GDR energy (19.3 MeV for the RPA). The experimental GDR width and energy are $6.98 \mathrm{MeV}$ and $19.5 \mathrm{MeV}$ [65], respectively. The calculated characteristics of the GDR are in agreement with the observed values. The general shapes of the GDR obtained in the PPC are rather close to those observed in experiment. This demonstrates the improvement of the PPC description in comparison with RPA. We conclude that the main mechanisms of the GDR formation in ${ }^{48} \mathrm{Ca}$ can be taken into account 

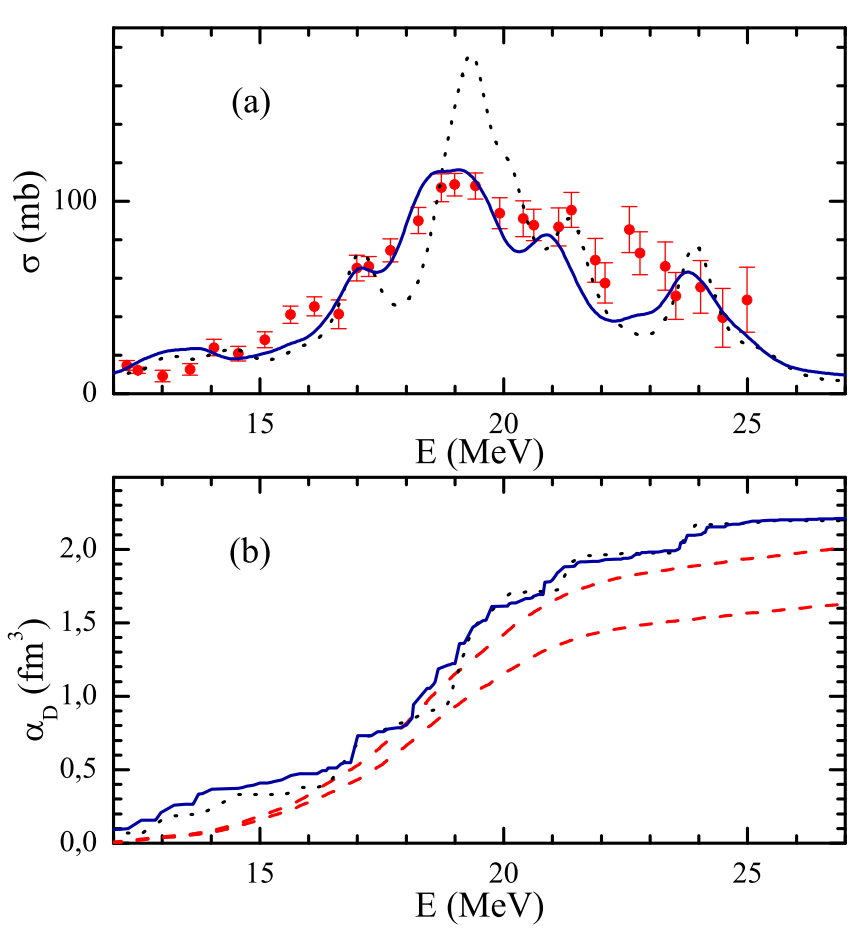

FIG. 5: (Color online) (a) The estimated photo-absorption cross section for ${ }^{48} \mathrm{Ca}$ (filled circles) are taken from Ref. 65]. The dotted and solid lines correspond to the calculations within the RPA with the SLy5 EDF and taking into account the PPC, respectively. (b) Running sum of the electric dipole polarizability for ${ }^{48} \mathrm{Ca}$ calculated within the RPA with the SLy5 EDF (dotted line) and the RPA plus PPC (solid line) in comparison to experimental determination of $\alpha_{D}$ (the two dashed lines indicate upper and lower limits) [52].

correctly and consistently in the PPC approach.

As proposed in Ref. [66], we can estimate the $M 1$ contribution to the photo-absorption process calculated in the case of the SLy5 EDF. For ${ }^{48} \mathrm{Ca}$, we find that the $M 1$ contribution plays a minor role $(<1 \%)$ for the integrated cross section. It was shown in the experimental papers [12, 13] that the contribution of $1^{+}$components to the total dipole strength below $10 \mathrm{MeV}$ is negligible. For ${ }^{44} \mathrm{Ca}$, the contribution of $M 1$ in the region of $3-10 \mathrm{MeV}$ to the total dipole strength is less than $3 \%$ [13]. In the GDR energy region, the $M 1$ contribution was zero within error bars and was replaced by a phenomenological background in the case of ${ }^{208} \mathrm{~Pb}[67]$. Thus, the magnetic counterparts have been omitted in our analysis.

In order to perform further investigations on the ${ }^{48} \mathrm{Ca}$ nucleus we have extracted the electric dipole polarizability [1, 68, 69], which represents a handle to constrain the equation of state of neutron matter and the physics of neutron stars [9, 10]. The electric dipole polarizability $\alpha_{D}$ is written as

$$
\alpha_{D}=\frac{8 \pi}{9} \sum_{\mu_{\alpha} \mu} \sum_{i_{\alpha}} E_{1_{i_{\alpha}}^{-}}^{-1}\left[\left\langle 1_{i_{\alpha} \mu_{\alpha}}^{-}\left|\hat{M}^{1 \mu}\right| 0_{g s}^{+}\right\rangle\right]^{2},
$$

where

$$
\hat{M}^{1 \mu}=-\frac{Z}{A} e \sum_{k=1}^{N} r_{k} Y_{1 \mu}\left(\hat{r}_{k}\right)+\frac{N}{A} e \sum_{k=1}^{Z} r_{k} Y_{1 \mu}\left(\hat{r}_{k}\right) .
$$

Here, $N, Z$, and $A$ are the neutron, proton, and mass numbers, respectively; $r_{k}$ indicates the radial coordinate for neutrons (protons); and $Y_{1 \mu}\left(\hat{r}_{k}\right)$ is the corresponding spherical harmonic. The definition of the dipole operator eliminates contaminations of the physical response due to the spurious excitation of the center of mass. In Ref. 70] it has been shown that eliminating the spurious state by means of effective charges or the alternative ways lead to very similar results.

Running sums of $\alpha_{D}$ values for ${ }^{48} \mathrm{Ca}$ in the energy region below $27 \mathrm{MeV}$ are given in Fig. 5 (b). It is shown that the PPC does not affect the description of the electric dipole polarizability. The results differ insignificantly. Moreover, we have checked that inclusion of the tensor components does not change the value of $\alpha_{D}$ obtained by integrating the $E 1$ strength up to $60 \mathrm{MeV}: \alpha_{D}=2.28 \mathrm{fm}^{3}$ in the case of the SLy5 EDF and $\alpha_{D}=2.20 \mathrm{fm}^{3}$ in the SLy5+T. Both effective interactions reproduce the experimental data $\alpha_{D}=2.07 \pm 0.22 \mathrm{fm}^{3}[52]$ and they are in good agreement with the "model-averaged" value of $2.306 \pm 0.089 \mathrm{fm}^{3}$, which is predicted in Ref. [53]. Although the GDR strength dominates, contributions to $\alpha_{D}$ value at lower and higher excitation energies must be taken into account.

To complete the discussion we consider $\alpha_{D}$ as a function of the neutron number for $\mathrm{Ca}$ isotopes, see Fig. 6 . The result of the SLy5 calculation with the PPC predicts a monotonic increase of the $\alpha_{D}$ value with neutron number, and only a small kink in the calculated excitation energies is found at the $N=28$ shell closure. The calculated polarizabilities $\alpha_{D}$ of ${ }^{40} \mathrm{Ca}$ and ${ }^{48} \mathrm{Ca}$ are in excellent agreement with the experimental data [52]. As shown in Fig. 6(a), the SLy5 and SLy5+T EDF's produce qualitatively the same results. We find that the correlation between the value of $\alpha_{D}$ and neutron skin $\Delta R_{n p}$ is discerned, see Fig. 6(b). With the increase of the neutron skin one observes a smooth increase of $\alpha_{D}$. Thus, the $\alpha_{D}$ value and the neutron skin $\Delta R_{n p}$ are correlated as predicted in Ref. 69]. Besides this, the $\alpha_{D}$ value can be measured in finite nuclei and, as a result, the $\Delta R_{n p}$ value can be extracted.

For ${ }^{48-58} \mathrm{Ca}$, we recognize the PDR energy below $10 \mathrm{MeV}$. The difference of the structure of the $1^{-}$states below and above $10 \mathrm{MeV}$ is illustrated with the QRPA transition densities in the cases of ${ }^{50} \mathrm{Ca}$ and ${ }^{56} \mathrm{Ca}$, see Fig. 7. For the $1^{-}$states below $10 \mathrm{MeV}$, the proton and neutron densities in the nuclear interior region are in phase. However one can see the neutron dominance when $r>6 \mathrm{fm}$. For the $1^{-}$states about $10 \mathrm{MeV}$, the corresponding transition proton and neutron densities are very similar to each other (see Fig. 7). These states can be identified as an isoscalar mode in the low-energy $E 1$ strength. This is not the PDR where the neutron skin oscillates against the inner core. The next fairly collective 

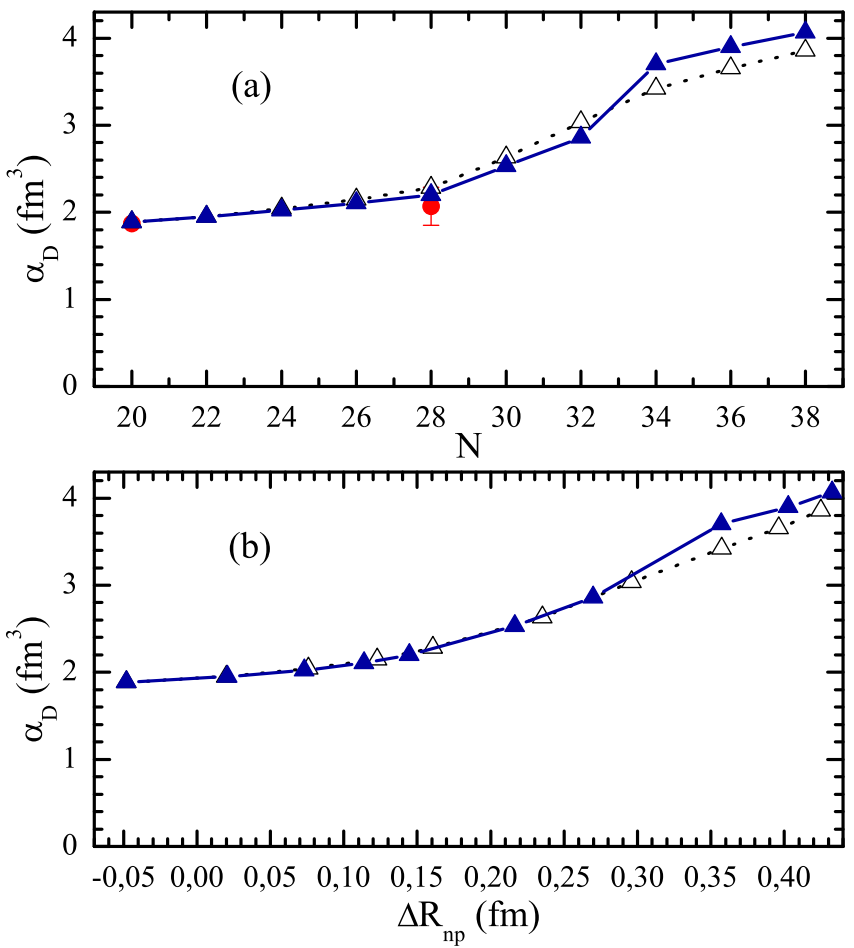

FIG. 6: (Color online) (a) The electric dipole polarizability $\alpha_{D}$ as a function of neutron number, calculated with the SLy5 EDF (open triangles) and with SLy5+T (filled triangles). Experimental values (filled circles) of $\alpha_{D}$ are taken from [52]. (b) The same as (a) but as a function of the neutron skin thickness $\Delta R_{n p}$.
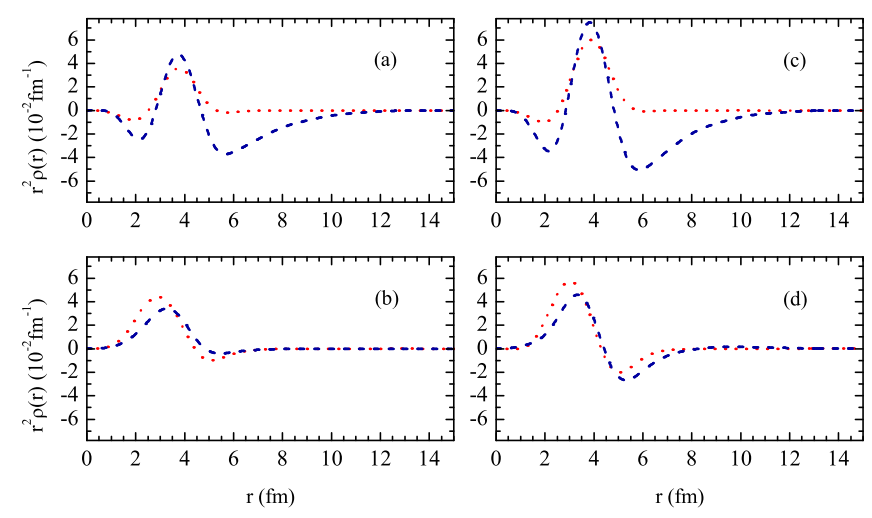

FIG. 7: (Color online) Transition proton (dotted line) and neutron (dashed line) densities to selected QRPA $1^{-}$states of ${ }^{50} \mathrm{Ca}$ (resp. ${ }^{56} \mathrm{Ca}$ ) in the left (resp. right) panels. Panels (a) and (b) correspond to the transition densities for the states at 9.1 MeV and $10.3 \mathrm{MeV}$ in ${ }^{50} \mathrm{Ca}$, while panels (c) and (d) show the transition densities for the QRPA $1^{-}$states at $8.7 \mathrm{MeV}$ and $10.7 \mathrm{MeV}$ in ${ }^{56} \mathrm{Ca}$, respectively. All transition densities are multiplied by $r^{2}$.

$1^{-}$state at $12.2 \mathrm{MeV}$ in ${ }^{50} \mathrm{Ca}$ and $12.1 \mathrm{MeV}$ in ${ }^{56} \mathrm{Ca}$ has neither the isoscalar character nor the isovector character. Increasing further the excitation energy we observe the low-energy GDR tail. Note that the PDR energy re-
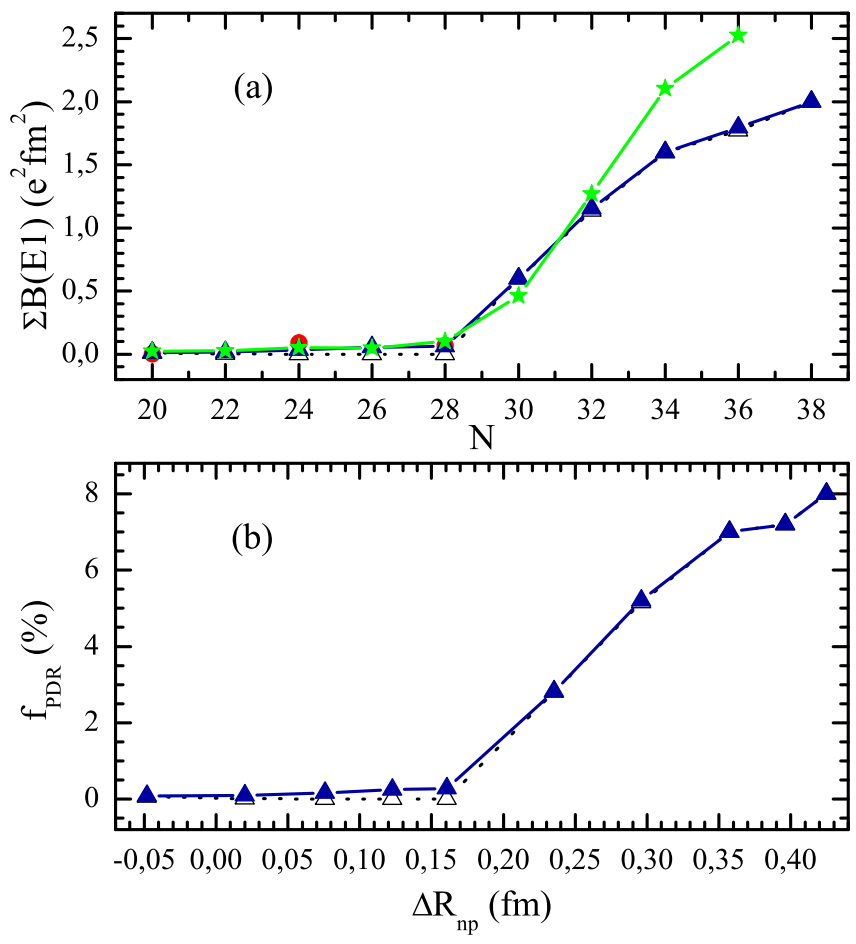

FIG. 8: (Color online) (a) Summed dipole strength below $10 \mathrm{MeV}$ calculated in the QRPA (open triangles) with the SLy5 EDF, PPC (filled triangles) and with RQTBA (filled stars) [17]. Experimental data (filled circles) are taken from Refs. [12]. (b) Ratio of the PDR energy-weighted strength to the TRK sum rule for various $\mathrm{Ca}$ isotopes as a function of the neutron skin thickness $\Delta R_{n p}$. The open triangles indicate discrete QRPA results, while the filled triangles are PPC results.

gion for the neutron-rich $\mathrm{Ca}$ isotopes has been found in the framework of other theoretical approaches [6, 17, 71].

Let us now discuss the low-energy $E 1$ strength. The collectivity of the PDR can be studied by plotting the evolution of its summed strength $\sum B(E 1)$ with respect to the mass number. The calculated PDR is integrated up to $10 \mathrm{MeV}$. As shown in the upper panel of Fig. 8, the behavior of the PDR summed strength can be divided into two categories: light and heavy $\mathrm{Ca}$ isotopes. In particular, we find that there is a sharp increase after the doubly magic isotope ${ }^{48} \mathrm{Ca}$ in the QRPA with the SLy5 EDF. In light Ca isotopes a completely different behavior is observed in Ref. [12]: the measured summed strength in ${ }^{44} \mathrm{Ca}$ is 18 times larger than in ${ }^{40} \mathrm{Ca}$ and 1.3 times in the case of ${ }^{48} \mathrm{Ca}$. From Fig. $8(\mathrm{a})$, one can see that the QRPA calculations fail to reproduce the experimental data. This result is in agreement with the nonrelativistic [16] and relativistic QRPA 71] calculations. Thus, the correlation between the PDR integrated strength and the neutron excess of $\mathrm{Ca}$ isotopes is nontrivial and it is necessary to go beyond QRPA to explain the properties of the PDR.

Let us now discuss the strong increase of the summed $E 1$ strength below $10 \mathrm{MeV}\left(\sum B(E 1)\right)$, with increasing 

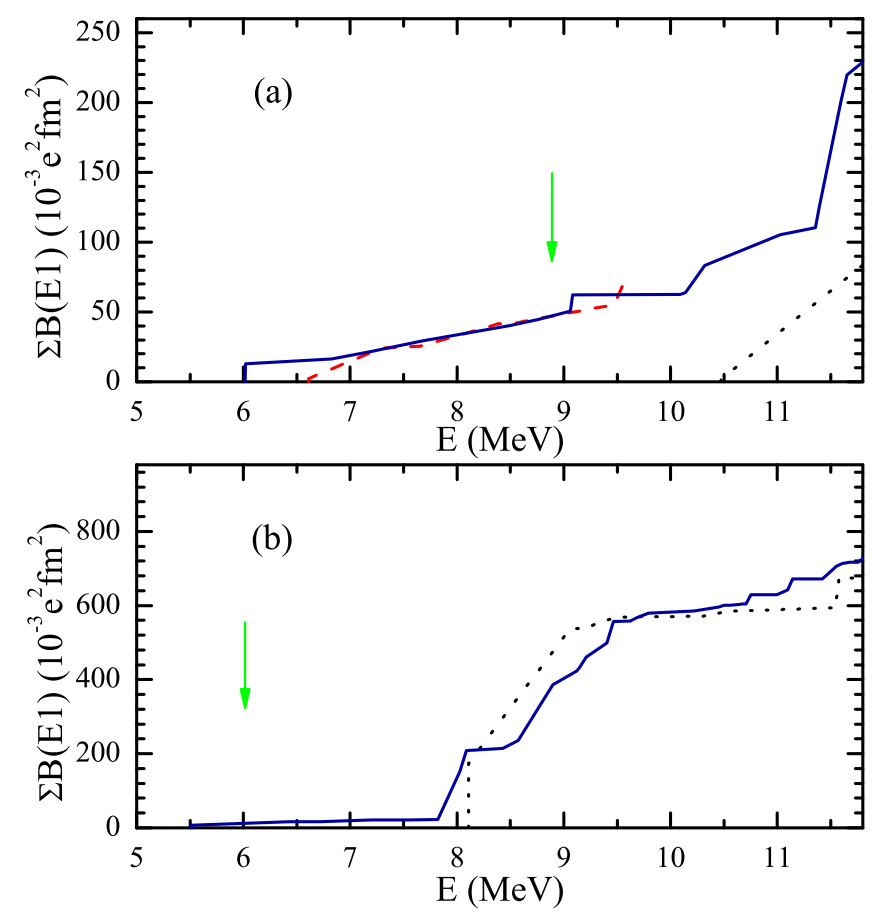

FIG. 9: (Color online) Running sums of the low-lying dipole strengths in ${ }^{48} \mathrm{Ca}$ (top panel) and ${ }^{50} \mathrm{Ca}$ (lower panel). The dotted and solid lines correspond to the calculations within the QRPA with the SLy5 EDF and taking into account the PPC, respectively. Experimental data (dashed line) are taken from Ref. [12]. The calculated $S_{1 n}$ energy is indicated by the solid arrow.

neutron number from ${ }^{48} \mathrm{Ca}$ till ${ }^{58} \mathrm{Ca}$. In Fig. 9(a) the calculated running sum for ${ }^{48} \mathrm{Ca}$ is plotted as a function of the excitation energy. In the same plot the calculated $S_{1 n}$ values are shown. In the case of the RPA, there is no $1^{-}$state below $10 \mathrm{MeV}$, see Fig. 4. The RPA calculations predict the first dipole state around $10.5 \mathrm{MeV}$. In contrast to the RPA case, the PPC results in the formation of low-lying $1^{-}$states in this energy region. The dominant contribution in the wave function of the $1^{-}$states comes from the two-phonon configurations $(>60 \%)$. These states originate from the fragmentation of the RPA states above $10 \mathrm{MeV}$. As one can see in Fig. 9(a), the calculated running sum of the $\sum B(E 1)$ value is close to the experimental $\sum B(E 1)$ value. The $\mathrm{PPC}$ calculations give a total dipole strength of $0.063 \mathrm{e}^{2} \mathrm{fm}^{2}$. The experimental value of $\sum B(E 1)$ is $0.0687 \pm 0.0075 \mathrm{e}^{2} \mathrm{fm}^{2}$ in the same interval [12]. The PPC effects produce a sizable impact on the low-energy $E 1$ strength of ${ }^{48} \mathrm{Ca}$. It is remarkable that the contributions of the low-lying $1^{-}$states to the value of $\alpha_{D}$ is small $\left(0.033 \mathrm{fm}^{3}\right)$, three times as much as the value deduced experimentally [52]. It is shown that the $\alpha_{D}$ value is more sensitive to the fine structure of the $E 1$ strength distribution. The PPC calculations reproduce the observed trend in light Ca isotopes, although the theoretical value of $\sum B(E 1)$ for ${ }^{44} \mathrm{Ca}$ underestimates the experimental value by a factor of 2 . It is worth men-
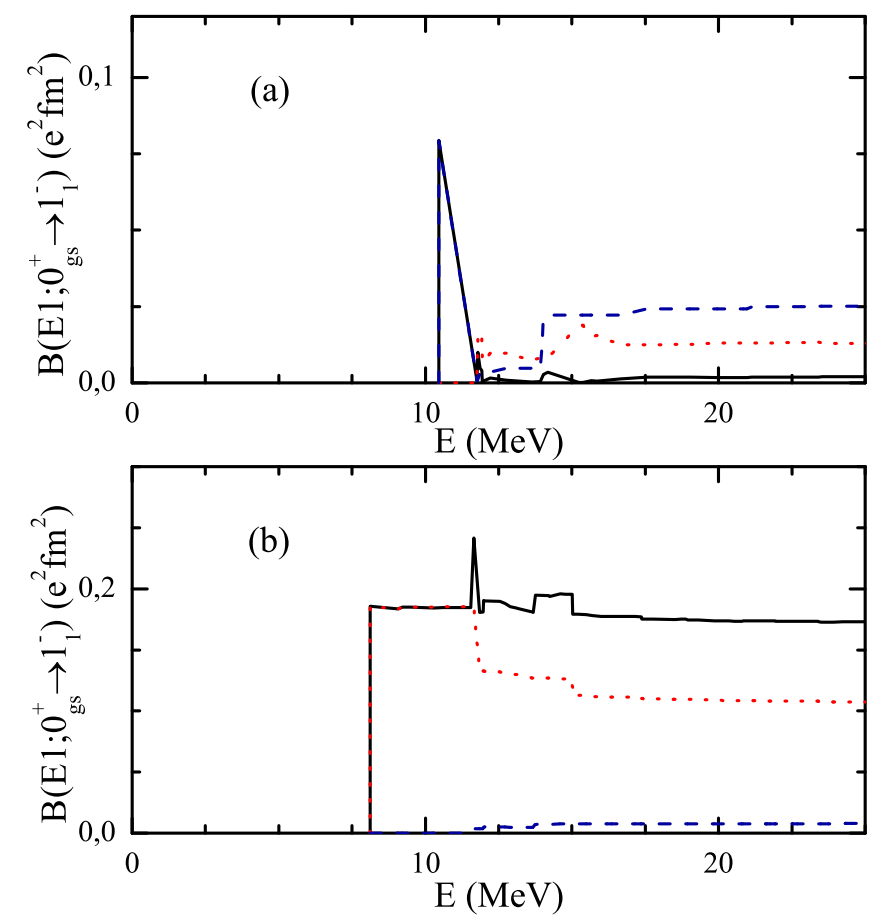

FIG. 10: (Color online) Running sums (solid lines) of the $B\left(E 1 ; 0_{g s}^{+} \rightarrow 1_{1}^{-}\right)$as a function of the two-quasiparticle energy included in the QRPA calculations for ${ }^{48} \mathrm{Ca}$ (top panel) and ${ }^{50} \mathrm{Ca}$ (lower panel). Dashed and dotted lines correspond to the results for the proton and the neutron components of the dipole operator (9), respectively.

tioning that the relativistic quasiparticle time blocking approximation (RQTBA) [17], the value of $\sum B(E 1)$ is also substantially less than the experimental one. As shown in Fig. 8(a), the two models produce qualitatively the same results.

Moving from ${ }^{48} \mathrm{Ca}$ to ${ }^{50} \mathrm{Ca}$, the QRPA calculations predict a jump of the $\sum B(E 1)$ value. $N=30$ corresponds to the occupation of $\nu 2 p \frac{3}{2}$ subshell, resulting in the two rather pronounced states below $10 \mathrm{MeV}$ being pure neutron 2QP excitations: $99 \%\left\{3 s \frac{1}{2} 2 p \frac{3}{2}\right\}_{\nu}$ and $98 \%\left\{2 d \frac{5}{2} 2 p \frac{3}{2}\right\}_{\nu}$. The summed $E 1$ strength of these states is $0.54 \mathrm{e}^{2} \mathrm{fm}^{2}$. As can be seen from Fig. 9(b), two states determine the value of $\sum B(E 1)$ calculated below $10 \mathrm{MeV}$. There is no contribution from the 2QP proton excitations. This structure is very different from that of the first $1^{-}$state in ${ }^{48} \mathrm{Ca}$, where the leading proton 2QP configuration $\left\{2 p \frac{3}{2} 1 d \frac{3}{2}\right\}_{\pi}$ gives a contribution of $96 \%$. For ${ }^{48} \mathrm{Ca}$, the closure of the neutron subshell $\nu 1 f \frac{7}{2}$ leads to the vanishing of the neutron pairing.

Fig. 10 shows the running sums for the QRPA value of $B\left(E 1 ; 0_{g s}^{+} \rightarrow 1_{1}^{-}\right)$as a function of the proton and neutron $2 \mathrm{QP}$ energies. In the case of ${ }^{48} \mathrm{Ca}$, the $B\left(E 1 ; 0_{g s}^{+} \rightarrow 1_{1}^{-}\right)$ is exhausted by the proton $2 \mathrm{QP}$ configurations, while the largest part of the $B(E 1)$ value for ${ }^{50} \mathrm{Ca}$ is generated by neutron excitations in the low-energy region. For ${ }^{50} \mathrm{Ca}$, the proton and neutron components contribute coher- 
ently. The main difference between these isotopes is that the neutron 2QP configurations contribute more than proton ones. This competition is mainly responsible for the $\sum B(E 1)$ increase.

In contrast to the case of ${ }^{48} \mathrm{Ca}$, the $\mathrm{PPC}$ effects on the low-energy dipole spectrum of ${ }^{50} \mathrm{Ca}$ is weak (see Fig. 9(b)). Thus, the $\sum B(E 1)$ values for ${ }^{50} \mathrm{Ca}$ results predominantly from the QRPA distribution of $E 1$ strength. As can be seen in Fig. 8(a) a similar result is observed in the case of ${ }^{52,54,56,58} \mathrm{Ca}$ isotopes. The separation energies decrease much faster than the value of $\sum B(E 1)$. This means, of course, that the observation of the PDR in $\left(\gamma, \gamma^{\prime}\right)$ experiments will be strongly hindered.

Let us now examine the correlation between the PDR properties and the neutron skin $\Delta R_{\mathrm{np}}$. To quantify the low-energy $E 1$ strength in a systematic analysis, we use the PDR fraction,

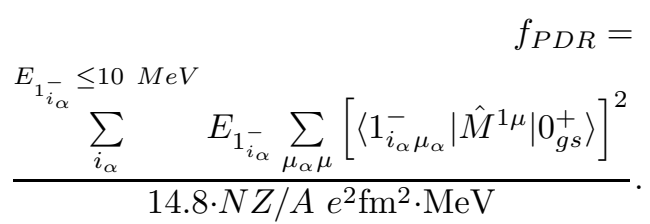

One of the basic ingredients for the fitting protocol of the SLy5 EDF is the enhancement factor of the TRK sum rule $\kappa=0.25$ [49]. Therefore, the total dipole strength exhausts $125 \%$ of the TRK sum rule, i.e., 18.5 $\mathrm{NZ} / A e^{2} \mathrm{fm}^{2} \cdot \mathrm{MeV}$. In Fig. 8(b) the $\Delta R_{\mathrm{np}}$ dependence of the $f_{P D R}$ for Ca isotopes is shown. The filled triangles indicate the PPC effects, which can be compared with QRPA results indicated by the open triangles. The ${ }^{48} \mathrm{Ca}\left(\gamma, \gamma^{\prime}\right)$ experiments give $f_{P D R}=0.33 \pm 0.04 \%$ [12], while the calculations with the PPC effects lead to $0.28 \%$ (to be compared with the RQTBA result of $0.55 \%$ [17]). The QRPA calculations predict that the visible $f_{P D R}$ kink can be identified at the magic neutron number $N=28$. The PDR fractions suddenly increase from $N=28$ to 30 and continue to increase until $N=34$ where the $\nu 2 p \frac{1}{2}$ subshell is filled. Beyond $N=34$, the neutrons start filling the $\nu 1 f \frac{5}{2}$ subshell, thus reducing the slope of $f_{P D R}$. The correlation between the $f_{P D R}$ and $\Delta R_{\mathrm{np}}$ turn out to be rather complex, depending on the neutron number.

\section{CONCLUSIONS}

The electric dipole polarizability is a particularly important observable, as it can be measured in finite nu- clei and it provides important information on the neutron skin thickness that can be extracted. In this study, Skyrme QRPA calculations including the phonon-phonon coupling have been performed for the $E 1$ response in neutron-rich $\mathrm{Ca}$ isotopes, some of which should become experimentally accessible in the near future. The FRSA enables one to perform the calculations in large configuration spaces. The SLy5 EDF and its modification including the tensor components SLy5+T reproduce the data for the neutron skin thickness and neutron separation energies. Among our initial motivation there was the estimation of dipole polarizability for ${ }^{50,52} \mathrm{Ca}$ in comparison to the $N=28$ isotope ${ }^{48} \mathrm{Ca}$. Our results describe the experimental data of ${ }^{40,48} \mathrm{Ca}$ and they give a sizable increase due to the neutron shell closure effect. It is shown that the phonon-phonon coupling has small influence on the dipole polarizability.

For ${ }^{48} \mathrm{Ca}$, the PPC effect have a damping and smoothing action which yields a GDR cross section close to the experimental one in shape and magnitude. We find the impact of the shell closure $N=28$ on the summed E1 strength below $10 \mathrm{MeV}$. There is also the $64 \%$ decrease of one-neutron separation energy from ${ }^{48} \mathrm{Ca}$ to ${ }^{50} \mathrm{Ca}$. This is due to the pairing effect on neutron $\nu 2 p \frac{3}{2}$ subshell for ${ }^{50} \mathrm{Ca}$. The dipole response for ${ }^{52-58} \mathrm{Ca}$ is characterized by the fragmentation of the strength distribution and its spreading into the low-energy region.

The model can be extended by enlarging the variational space for the $1^{-}$states with the inclusion of the three-phonon configurations. The computational developments that would allow us to conclude on this point are underway.

\section{Acknowledgments}

We are thankful to I. A. Egorova, N. Pietralla, M. Scheck and Ch. Stoyanov for many fruitful and stimulating discussions concerning various aspect of this work. The authors are very thankful to E. O. Sushenok for help in calculations of the neutron separation energies. N.N.A., A.P.S., and V.V.V. thank the hospitality of IPNOrsay where the part of this work was done. This work is partly supported by the CNRS-RFBR agreement No. 1652-150003, the IN2P3-JINR agreement, and the RFBR grant No. 16-02-00228.
[1] A. B. Migdal, J. Phys. Acad. Sci. USSR 8, 331 (1944); J. Exp. Theor. Phys. USSR 15, 81 (1945).

[2] B. L. Berman and S. C. Fultz, Rev. Mod. Phys. 47, 713
(1975).

[3] S. S. Dietrich and B. L. Berman, At. Data Nucl. Data Tables 38, 199 (1988). 
[4] D. Savran, T. Aumann, and A. Zilges, Prog. Part. Nucl. Phys. 70, 210 (2013).

[5] N. Paar, D. Vretenar, E. Khan, and G. Colò, Rep. Prog. Phys. 70, 691 (2007).

[6] T. Inakura, T. Nakatsukasa, and K. Yabana, Phys. Rev. C 84, 021302(R) (2011).

[7] S. Ebata, T. Nakatsukasa, and T. Inakura, Phys. Rev. C 90, 024303 (2014); 92, 049902(E) (2015).

[8] M. Arnould, S. Goriely, and K. Takahashi, Phys. Rep. 450, 97 (2007).

[9] B. A. Brown, Phys. Rev. Lett. 85, 5296 (2000).

[10] C. J. Horowitz and J. Piekarewicz, Phys. Rev. Lett. 86, 5647 (2001).

[11] T. Hartmann, J. Enders, P. Mohr, K. Vogt, S. Volz, and A. Zilges, Phys. Rev. Lett. 85, 274 (2000); 86, 4981(E) (2001).

[12] T. Hartmann, M. Babilon, S. Kamerdzhiev, E. Litvinova, D. Savran, S. Volz, and A. Zilges, Phys. Rev. Lett. 93, 192501 (2004).

[13] J. Isaak, D. Savran, M. Fritzsche, D. Galaviz, T. Hartmann, S. Kamerdzhiev, J. H. Kelley, E. Kwan, N. Pietralla, C. Romig, G. Rusev, K. Sonnabend, A. P. Tonchev, W. Tornow, and A. Zilges, Phys. Rev. C 83, 034304 (2011).

[14] V. Derya, D. Savran, J. Endres, M. N. Harakeh, H. Hergert, J. H. Kelley, P. Papakonstantinou, N. Pietralla, V. Yu. Ponomarev, R. Roth, G. Rusev, A. P. Tonchev, W. Tornow, H. J. Wörtche, and A.Zilges, Phys. Lett. B 730, 288 (2014).

[15] V. G. Soloviev, Ch. Stoyanov, and V. V. Voronov, Nucl. Phys. A 304, 503 (1978).

[16] J. Terasaki and J. Engel, Phys. Rev. C 74, 044301 (2006).

[17] I. A. Egorova and E. Litvinova, Phys. Rev. C 94, 034322 (2016).

[18] A. Gade, R. V. F. Janssens, D. Bazin, R. Broda, B. A. Brown, C. M. Campbell, M. P. Carpenter, J. M. Cook, A. N. Deacon, D.-C. Dinca, B. Fornal, S. J. Freeman, T. Glasmacher, P. G. Hansen, B. P. Kay, P. F. Mantica, W. F. Mueller, J. R. Terry, J. A. Tostevin, and S. Zhu, Phys. Rev. C 74, 021302(R) (2006).

[19] F. Wienholtz, D. Beck, K. Blaum, Ch. Borgmann, M. Breitenfeldt, R. B. Cakirli, S. George, F. Herfurth, J. D. Holt, M. Kowalska, S. Kreim, D. Lunney, V. Manea, J. Menéndez, D. Neidherr, M. Rosenbusch, L. Schweikhard, A. Schwenk, J. Simonis, J. Stanja, R. N.Wolf, and K. Zuber, Nature 498, 346 (2013).

[20] D. Steppenbeck, S. Takeuchi, N. Aoi, P. Doornenbal, M. Matsushita, H. Wang, H. Baba, N. Fukuda, S. Go, M. Honma, J. Lee, K. Matsui, S. Michimasa, T. Motobayashi, D. Nishimura, T. Otsuka, H. Sakurai, Y. Shiga, P.-A. Söderström, T. Sumikama, H. Suzuki, R. Taniuchi, Y. Utsuno, J. J. Valiente-Dobón, and K. Yoneda, Nature 502, 207 (2013).

[21] O. B. Tarasov, D. J. Morrissey, A. M. Amthor, T. Baumann, D. Bazin, A. Gade, T. N. Ginter, M. Hausmann, N. Inabe, T. Kubo, A. Nettleton, J. Pereira, M. Portillo, B. M. Sherrill, A. Stolz, and M. Thoennessen, Phys. Rev. Lett. 102, 142501 (2009).

[22] V. G. Soloviev, Theory of Atomic Nuclei: Quasiparticles and Phonons (Institute of Physics, Bristol and Philadelphia, 1992).

[23] N. Lo Iudice, V. Yu. Ponomarev, Ch. Stoyanov, A. V. Sushkov, and V. V. Voronov, J. Phys. G 39, 043101 (2012).
[24] M. Grinberg and Ch. Stoyanov, Nucl. Phys. A 573, 231 (1994).

[25] V.Yu. Ponomarev, Ch. Stoyanov, N. Tsoneva, and M. Grinberg, Nucl.Phys. A 635, 470 (1998).

[26] N. Tsoneva, H. Lenske, and Ch. Stoyanov, Phys. Lett. B 586, 213 (2004).

[27] N. Tsoneva and H. Lenske, Phys. Rev. C 77, 024321 (2008).

[28] N. Tsoneva, S. Goriely, H. Lenske, and R. Schwengner, Phys. Rev. C 91, 044318 (2015).

[29] D. Sarchi, P. F. Bortignon, and G. Colò, Phys. Lett. B 601, 27 (2004).

[30] A. Avdeenkov, S. Goriely, S. Kamerdzhiev, and S. Krewald, Phys. Rev. C 83, 064316 (2011).

[31] N. N. Arsenyev, A. P. Severyukhin, V. V. Voronov, and Nguyen Van Giai, EPJ Web of Conf. 38, 17002 (2012).

[32] A. Repko, P.-G. Reinhard, V. O. Nesterenko, and J. Kvasil, Phys. Rev. C 87, 024305 (2013).

[33] Nguyen Van Giai, Ch. Stoyanov, and V. V. Voronov, Phys. Rev. C 57, 1204 (1998).

[34] A. P. Severyukhin, Ch. Stoyanov, V. V. Voronov, and Nguyen Van Giai, Phys. Rev. C 66, 034304 (2002).

[35] A. P. Severyukhin, V. V. Voronov, and Nguyen Van Giai, Eur. Phys. J. A 22, 397 (2004).

[36] A. P. Severyukhin, V. V. Voronov, and Nguyen Van Giai, Phys. Rev. C 77, 024322 (2008).

[37] T. Suzuki and H. Sagawa, Prog. Theor. Phys. 65, 565 (1981).

[38] P. Sarriguren, E. Moya de Guerra, and A. Escuderos, Nucl. Phys. A 658, 13 (1999).

[39] V. O. Nesterenko, J. Kvasil, and P.-G. Reinhard, Phys. Rev. C 66, 044307 (2002).

[40] A. P. Severyukhin, N. N. Arseniev, V. V. Voronov, and Nguyen Van Giai, Phys. At. Nucl. 72, 1149 (2009).

[41] A. P. Severyukhin, N. N. Arsenyev, and N. Pietralla, Phys. Rev. C 86, 024311 (2012).

[42] N. N. Arsenyev, A. P. Severyukhin, V. V. Voronov, and Nguyen Van Giai, Acta Phys. Pol. B 46, 517 (2015).

[43] N. N. Arsenyev, A. P. Severyukhin, V. V. Voronov, and Nguyen Van Giai, EPJ Web of Conf. 107, 05006 (2016).

[44] P. Ring and P. Schuck, The Nuclear Many Body Problem (Springer, Berlin, 1980).

[45] F. Stancu, D. M. Brink, and H. Flocard, Phys. Lett. B 68, 108 (1977).

[46] T. Lesinski, M. Bender, K. Bennaceur, T. Duguet, and J. Meyer, Phys. Rev. C 76, 014312 (2007).

[47] J. Terasaki, J. Engel, M. Bender, J. Dobaczewski, W. Nazarewicz, and M. Stoitsov, Phys. Rev. C 71, 034310 (2005).

[48] A. P. Severyukhin, V. V. Voronov, I. N. Borzov, N. N. Arsenyev, and Nguyen Van Giai, Phys. Rev. C 90, 044320 (2014).

[49] E. Chabanat, P. Bonche, P. Haensel, J. Meyer, and R. Schaeffer, Nucl. Phys. A 635, 231 (1998); 643, 441(E) (1998).

[50] G. Colò, H. Sagawa, S. Fracasso, and P. F. Bortignon, Phys. Lett. B 646, 227 (2007); 668, 457(E) (2008).

[51] M. Wang, G. Audi, A. H. Wapstra, F. G. Kondev, M. MacCormick, X. Xu, and B. Pfeiffer, Chin. Phys. C 36, 1603 (2012).

[52] J. Birkhan, M. Miorelli, S. Bacca, S. Bassauer, C. A. Bertulani, G. Hagen, H. Matsubara, P. von Neumann-Cosel, T. Papenbrock, N. Pietralla, V. Yu. Ponomarev, A. Richter, A. Schwenk, and A. Tamii, 
arXiv:1611.07072v2 [nucl-ex].

[53] J. Piekarewicz, B. K. Agrawal, G. Colò, W. Nazarewicz, N. Paar, P.-G. Reinhard, X. Roca-Maza, and D. Vretenar, Phys. Rev. C 85, 041302(R) (2012).

[54] G. Hagen, A. Ekström, C. Forssén, G. R. Jansen, W. Nazarewicz, T. Papenbrock, K. A. Wendt, S. Bacca, N. Barnea, B. Carlsson, C. Drischler, K. Hebeler, M. HjorthJensen, M. Miorelli, G. Orlandini, A. Schwenk, and J. Simonis, Nature Physics 12, 186 (2016).

[55] V. G. Soloviev, Kgl. Dan. Vid. Selsk. Mat. Fys. Skr. 1, 238 (1961).

[56] J. Dobaczewski, W. Satuła, B. G. Carlsson, J. Engel, P. Olbratowski, P. Powałowski, M. Sadziak, J. Sarich, N. Schunck, A. Staszczak, M. Stoitsov, M. Zalewski, and H. Zduńczuk, Comput. Phys. Commun. 180, 2361 (2009).

[57] M. Grasso, Phys. Rev. C 89, 034316 (2014).

[58] J. D. Holt, T. Otsuka, A. Schwenk, and T. Suzuki, J. Phys. G 39, 085111 (2012).

[59] G. Hagen, M. Hjorth-Jensen, G. R. Jansen, R. Machleidt, and T. Papenbrock, Phys. Rev. Lett. 109, 032502 (2012).

[60] S.-C. Wu, Nucl. Data Sheets 91, 1 (2000).

[61] T. Kibédi and R. H. Spear, At. Data Nucl. Data Tables 80, 35 (2002).

[62] T. W. Burrows, Nucl. Data Sheets 107, 1747 (2006).

[63] D. Montanari, S. Leoni, D. Mengoni, J. J. ValienteDobon, G. Benzoni, N. Blasi, G. Bocchi, P. F. Bortignon, S. Bottoni, A. Bracco, F. Camera, P. Casati, G. Colò, A. Corsi, F. C. L. Crespi, B. Million, R. Nicolini, O.Wieland, D. Bazzacco, E. Farnea, G. Germogli, A. Gottardo, S. M. Lenzi, S. Lunardi, R. Menegazzo, G. Montagnoli, F. Recchia, F. Scarlassara, C. Ur, L. Corradi, G. de Angelis, E.
Fioretto, D. R. Napoli, R. Orlandi, E. Sahin, A. M. Stefanini, R. P. Singh, A. Gadea, S. Szilner, M. Kmiecik, A. Maj, W. Meczynski, A. Dewald, T. Pissulla, and G. Pollarolo, Phys. Rev. C 85, 044301 (2012).

[64] V. Derya, N. Tsoneva, T. Aumann, M. Bhike, J. Endres, M. Gooden, A. Hennig, J. Isaak, H. Lenske, B. Löher, N. Pietralla, D. Savran, W. Tornow, V. Werner, and A. Zilges, Phys. Rev. C 93, 034311 (2016).

[65] G. J. O'Keefe, M. N. Thompson, Y. I. Assafiri, R. E. Pywell, and K. Shoda, Nucl. Phys. A 469, 239 (1987).

[66] E. Lipparini and S. Stringari, Phys. Rep. 175, 103 (1989).

[67] A. Tamii, I. Poltoratska, P. von Neumann-Cosel, Y. Fujita, T. Adachi, C. A. Bertulani, J. Carter, M. Dozono, H. Fujita, K. Fujita, K. Hatanaka, D. Ishikawa, M. Itoh, T. Kawabata, Y. Kalmykov, A. M. Krumbholz, E. Litvinova, H. Matsubara, K. Nakanishi, R. Neveling, H. Okamura, H. J. Ong, B. Özel-Tashenov, V. Yu. Ponomarev, A. Richter, B. Rubio, H. Sakaguchi, Y. Sakemi, Y. Sasamoto, Y. Shimbara, Y. Shimizu, F. D. Smit, T. Suzuki, Y. Tameshige, J. Wambach, R. Yamada, M. Yosoi, and J. Zenihiro, Phys. Rev. Lett. 107, 062502 (2011).

[68] O. Bohigas, Nguyen Van Giai, and D. Vautherin, Phys. Lett. B 102, 105 (1981).

[69] W. Satuła, R. A. Wyss, and M. Rafalski, Phys. Rev. C 74, 011301(R) (2006).

[70] N. N. Arsenyev and A. P. Severyukhin, Phys. Part. Nucl. Lett. 7, 112 (2010).

[71] D. Vretenar, N. Paar, P. Ring, and G. A. Lalazissis, Nucl. Phys. A 692, 496 (2001). 